\title{
Efecto Del Almidón De Plátano Cuadrado Musa Balbisiana Colla En El Rendimiento, Retención De Agua Y Aceptación Sensorial En Jamón Cocid
}

\author{
Lázaro De La Torre-Gutiérrez (M of S) \\ Sandi Patricia Morales-Córdova (F. Eng.) \\ José Ulises González-de la Cruz (PhD) \\ María Concepción de la Cruz-Leyva (PhD) \\ División Académica Multidisciplinaria de los Ríos, Universidad Juárez \\ Autónoma de Tabasco,Tabasco, México
}

Doi:10.19044/esj.2021.v17n25p82

Submitted: 05 July 2021

Accepted: 10 July 2021

Published: 31 July 2021
Copyright 2021 Author(s)

Under Creative Commons BY-NC-ND 4.0 OPEN ACCESS

Cite As:

De La Torre-Gutiérrez L., Morales-Córdova S.P., González-de la Cruz J.U. \& Concepción de la Cruz-Leyva M.(2021). Effect Of Square Banana Musa Balbisiana Colla Starch On Yield, Water Retention And Sensory Acceptability In Cooked Ham. European Scientific Journal, ESJ, 17(25), 82.

https://doi.org/10.19044/esj.2021.v17n25p82

\section{Resumen}

Los almidones no convencionales se han utilizado con éxito en la formulación de productos cárnicos, al brindar textura y escaso sabor a un menor costo. Aquí el objetivo fue, evaluar el efecto del almidón de plátano cuadrado Musa balbisiana Colla sobre el rendimiento, retención de agua y aceptación sensorial en el jamón cocido. El almidón de plátano (madurez tipo I) se extrajo con una metodología recomendada. Se elaboró jamón cocido con carne de cerdo y se adicionó almidón de plátano. A partir de un diseño experimental factorial 22: concentración de almidón (factor A) 10 \% y 20 \%, y tiempo de masajeo de la carne (factor B) de 16 y 30 min. Resultando un total de cuatro tratamientos: T1, T2, T3 y T4 $(n=3)$. Para tener un referente, se elaboró un jamón cocido adicionado con almidón de maíz. La concentración de almidón de plátano y el mayor tiempo de masajeo (30 min), registró relación positiva en el rendimiento del jamón cocido (121.4 $\pm 0.36 \%$ ). La capacidad de retención de agua aumentó de acuerdo con la concentración de almidón y el tiempo de masajeo, independiente del origen del ligante 
adicionado (almidón de plátano o almidón de maíz). El jamón cocido adicionado con almidón de plátano fue el más aceptado y mejor evaluado. Por lo que, este almidón puede ser utilizado como un aditivo alimentario para la producción del jamón cocido.

Palabras Clave: Agente Ligante, Almidón De Plátano, Cárnicos

\title{
Effect Of Square Banana Musa Balbisiana Colla Starch On Yield, Water Retention And Sensory Acceptability In Cooked Ham
}

\author{
Lázaro De La Torre-Gutiérrez (M of S) \\ Sandi Patricia Morales-Córdova (F. Eng.) \\ José Ulises González-de la Cruz (PhD) \\ María Concepción de la Cruz-Leyva (PhD) \\ División Académica Multidisciplinaria de los Ríos, Universidad Juárez \\ Autónoma de Tabasco,Tabasco, México
}

\begin{abstract}
Non-conventional starches have been successfully used in the formulation of meat products, providing texture and low flavor at a lower cost. The objective here was to evaluate the effect of square banana Musa balbisiana Colla starch on yield, water retention and sensory acceptability in cooked ham. Banana starch (maturity type I) was extracted using a recommended methodology. Cooked ham (with pork meat) was prepared with banana starch. Based on a 22 factorial experimental design: starch concentration (factor A) $10 \%$ and $20 \%$, and meat massaging time (factor B) of 16 and $30 \mathrm{~min}$, resulting in a total of four treatments: T1, T2, T3 and T4 $(\mathrm{n}=3)$. In order to have a reference, a cooked ham was prepared with corn starch added. The concentration of banana starch and the longer massaging time (30 min) were positively related to the yield of cooked ham (121.4 $\pm 0.36 \%)$. Water retention capacity increased according to starch concentration and massaging time, independent of the origin of the added binder (banana starch or cornstarch). The cooked ham added with banana starch was the most accepted and best evaluated. Therefore, this starch can be used as a food additive to produce cooked ham.
\end{abstract}

Key Words: Binding Agent, Banana Starch, Meat Products 


\section{Introducción}

Los países con mayor consumo de productos cárnicos son: China (27 \%), Estados Unidos (16 \%), Unión Europea (15 \%), Brasil (8 \%), Rusia (4 \%) y México (3\%) (Compendio Estadístico, 2021). En nuestro país este sector representa el $23 \%$ de la industria alimentaria y el $32 \%$ del producto interno bruto (PIB) agropecuario (Porcicultura.com, 2020). El 40 \% de los cárnicos son jamones, donde el 55 \% se fabrica con carne de cerdo (Compendio Estadístico, 2021). Los productos cárnicos son importantes en la dieta humana, al proporcionar nutrientes como las proteínas, que no se obtiene fácilmente de las verduras y sus derivados (Alao et al., 2017). Coadyuvan para disminuir la malnutrición y aumentar la seguridad alimentaria (Tofiño-Rivera et al., 2017).

El jamón es el embutido hecho con carne de cerdo o pavo tras retirar los huesos; se aplica una salmuera con cloruro de sodio, nitrato y nitrito sódico, que inhiben el crecimiento de bacterias en la carne. En algunos casos, se agrega carragenina (1.5\%), fécula (5 \% a $10 \%$ ) y soya, que proporcionan textura, cuerpo y proteínas (2 \%). Se disminuye el costo del producto, al utilizar menor concentración de carne (PROFECO, 2016). En su caso, el jamón cocido se prepara con carne de las patas traseras del cerdo (pernil de cerdo), generalmente contiene: sacarosa, azúcar invertido, dextrosa (glucosa), lactosa, maltosa, miel, especias y gelatina (CODEX STAN 96-1981). En la etapa final de su elaboración se somete a $70{ }^{\circ} \mathrm{C}$ en el centro de la pieza para su cocción por inmersión y quedar listo para su consumo (NOM-213-SSA12018).

Algunas características deseables en el jamón cocido es el rendimiento: relación entre la materia prima utilizada y el producto obtenido; al igual que, el contenido de proteínas (16.5 \%) (CODEX STAN 96-1981). Es importante citar que el tiempo de masajeo es primordial para una distribución homogénea de la salmuera en la carne y una correcta extracción proteica. Entre las alternativas para mantener el bajo costo de producción, surgió el uso de ligantes o rellenos en los productos cárnicos (Tofiño-Rivera et al., 2017). Los ligadores son sustancias que tienen la función de ocupar el lugar de la carne, uniendo una cantidad considerable de agua, pero sin un aporte proteico (Jaimes et al., 2015). Un ejemplo de ello es el almidón nativo proveniente del trigo, maíz, arroz, papa, yuca, entre otros, son una fuente de aditivos naturales procedentes de zonas tropicales con un mínimo valor comercial. El almidón es un polímero de glucosa que sirve como reserva de energía en plantas superiores (Hernández et al., 2017).

Los almidones o féculas de materias primas no convencionales como el plátano, raíces, tubérculos y frutas tropicales no son muy solicitadas por los consumidores, debido al poco conocimiento de sus propiedades (Hernández et al., 2017). Sin embargo, se ha investigado una variedad de aplicaciones para 
el almidón con respecto a las industrias alimentarias y no alimentarias (Ssonko \& Muranga, 2017).

Bohórquez et al., (2017) evaluaron el almidón de raíz de achira Canna edulis Ker, como ingrediente en la formulación de alimentos; donde demostraron el uso potencial como estabilizante, gelificante, espesante y pastas.

Marrugo-Ligardo et al., (2017) elaboraron salchicha con diferentes niveles de almidón modificado de frijol Phaseolus lunatus. Indicaron que los niveles de almidón no afectaron la calidad nutricional de las salchichas y no encontraron diferencias significativas en el análisis del perfil de textura.

Se ha citado que la harina de plátano verde puede ser utilizada como sustituto comercial para producir galletas sin gluten con alto contenido de fibra dietética y bajo índice glucémico (García-Solis et al., 2018). Este tipo de almidón ha demostrado buenos resultados en la industria alimentaria, convirtiéndose en un aditivo alternativo para el desarrollo de productos como salsas, sopas, pates, entre otros (Fontes et al., 2017).

En cuanto a la composición química de la harina y el almidón nativo del plátano cuadrado en el estado fisiológico verde se ha detectado: $4.4 \% \pm$ 0.4 y $1.19 \% \pm 0.04$ de proteína cruda respectivamente; $2.44 \% \pm 0.01$ y 0.16 \pm 0.01 cenizas respectivamente. Así como, $0.85 \% \pm 0.22$ y $0.56 \pm 0.14$ extracto etéreo respectivamente (Contreras-Pérez et al., 2018). Por lo anterior, el objetivo fue evaluar el efecto del almidón de plátano cuadrado Musa balbisiana Colla en el rendimiento, retención de agua y aceptación sensorial en jamón cocido, como alternativa de un agente ligante para embutidos emulsionados.

\section{Materiales y Métodos \\ Materia prima}

Los frutos de M. balbisiana Colla con madurez tipo I (plátanos verde) se adquirieron en el mercado público local, ubicado en Tenosique, Tabasco México. Las piernas de cerdo se consiguieron en un establecimiento comercial (Maxi-Carne ${ }^{\circledR}$ ), esta tenía un pH de 5.7. También se adquirió almidón de maíz $\left(\right.$ Maizena $^{\circledR}$ ) para establecer un tratamiento comparativo.

\section{Extracción del almidón}

La extracción del almidón de los frutos de M. balbisiana C, se realizó mediante la metodología indicada por Contreras-Pérez et al., (2018) y ChelGuerrero et al., (2016).

\section{Elaboración de jamón cocido}

Se estableció un diseño experimental factorial general de dos factores con dos niveles cada uno $\left(2^{2}\right)$ : concentración de almidón (factor A) en dos 
niveles de concentración de almidón (10 \% y 20 \%) y tiempo de masajeo de la carne (factor B), durante dos tiempos (16 y $30 \mathrm{~min}$ ). Las variables respuesta fueron: rendimiento del jamón, capacidad de retención de agua y aceptación sensorial.

El pernil de cerdo se deshuesó, se limpió (se eliminó: grasa, nervios y aponeurosis) y la carne se cortó en porciones de aproximadamente $5 \mathrm{~cm}$. Se dejó en refrigeración hasta alcanzar una temperatura de $4 \pm 1^{\circ} \mathrm{C}$.

El jamón se elaboró según el procedimiento indicado en la bibliografía (Toledo, 2019; Luna \& Guerrero-Beltrán, 2013). Donde se adicionó almidón de plátano cuadrado $M$. balbisiana para observar la capacidad de retención de agua y aceptación sensorial. Se elaboró un jamón adicionado con almidón de maíz comercial (Maizena ${ }^{\circledR}$ ).

En cada caso (jamón con almidón de plátano o jamón con almidón de maíz) se establecieron cuatro tratamientos ( $\mathrm{n}=3$ ). Tratamiento 1 (T1): $10 \%$ de almidón y 16 min de masajeo. Tratamiento 2 (T2): 20 \% de almidón y 16 min de masajeo. Tratamiento 3 (T3): $10 \%$ de almidón y 30 min de masajeo. Tratamiento 4 (T4): 20 \% de almidón y 30 min de masajeo.

En todos los tratamientos se utilizó salmuera básica compuesta por: agua (95.35\%), nitrito de sodio (0.8\%), cloruro de sodio (2.2\%), fosfato de sodio (0.5\%) como estabilizante, eritorbato de sodio (0.3\%) como antioxidante, sacarosa $(0.8 \%)$ y humo líquido $(0.05 \%)$.

La temperatura de la salmuera fue de $2{ }^{\circ} \mathrm{C}$ durante su aplicación. Los jamones se envasaron con la ayuda de un equipo de vacío y se conservaron en refrigeración $\left(5^{\circ} \mathrm{C}\right)$ hasta su análisis.

\section{Rendimiento del jamón}

El rendimiento del jamón se estimó mediante el cálculo de la diferencia de peso del producto antes y después de la cocción; el resultado se expresó en porcentaje (Julio et al., 2015).

\section{Capacidad de retención de agua}

Para evaluar la capacidad de retención de agua (CRA), se utilizó una modificación de la metodología reportada por Braña et al., (2011).

Se depositó $5 \mathrm{~g}$ de carne en tubos de centrífuga graduados, por duplicado. Se adicionó $8 \mathrm{~mL}$ de solución fría de $\mathrm{NaCl} 0.6 \mathrm{M}$ y se agitó con una varilla de vidrio por 1 minuto. Posteriormente, se colocaron los tubos en un baño de hielo durante 30 minutos. Se agitó con una varilla de vidrio durante un minuto. Nuevamente, se centrifugaron los tubos por $15 \mathrm{~min}$ a 10,000 rpm y $4{ }^{\circ} \mathrm{C}$; se decantaron y se midió a todos el sobrenadante. 


\section{Evaluación sensorial}

Se utilizó un panel de jueces no entrenados integrado por 80 estudiantes universitarios. Se realizó un análisis de aceptación, preferencia y nivel de agrado mediante una escala hedónica estructurada de cinco puntos (Luna \& Guerrero-Beltrán, 2013).

\section{Análisis estadístico}

Utilizando el programa Statgraphics versión 16.1 se desarrolló un ANOVA y se evaluó la existencia de diferencia en medias Tukey con un nivel de significancia de $\mathrm{P}<0,05$. Posteriormente, se realizó una comparación medias por medio de LSD (diferencia mínima significativa) para determinar el mejor tratamiento en relación con el rendimiento. El efecto ligante se describió con una gráfica de superficie de respuestas. Los resultados del análisis sensorial se analizaron con el método de distribución binomial, según la tabla mínimo número de respuestas correctas para establecer significancia a diferentes niveles de probabilidad.

\section{Resultados y Discusión Rendimiento del jamón}

El análisis estadístico de los resultados del rendimiento del jamón cocido adicionado con almidón de plátano o el jamón cocido adicionado con almidón de maíz, no mostró un efecto significativo ( $\mathrm{P}>0.05)$ a un tiempo de masajeo de 16 minutos e independiente de la concentración de almidón (10 \% o $20 \%$ ).

No obstante, entre ambos tipos de almidón se registró un mayor rendimiento en los jamones adicionados con almidón de plátano cuadrado verde. El tratamiento T3 (10 \% de almidón) presentó un rendimiento de 120.6 \pm 0.15 y el tratamiento T4 ( $20 \%$ de almidón) registró un rendimiento de 121.4 \pm 0.36 asociados a mayor tiempo de masajeo de la carne (30 min) (Cuadro 1).

Cuadro 1. Rendimiento de jamón cocido adicionado con almidón de plátano o almidón de maíz

\begin{tabular}{|l|l|l|l|l|}
\hline Tratamiento & $\begin{array}{l}\text { Almidón } \\
(\mathbf{\% )})\end{array}$ & $\begin{array}{l}\text { Tiempo de } \\
\text { masajeo } \\
\text { (min)** }\end{array}$ & $\begin{array}{l}\text { Jamón adicionado } \\
\text { con almidón de } \\
\text { plátano (\%) }\end{array}$ & $\begin{array}{l}\text { Jamón adicionado } \\
\text { con almidón de } \\
\text { maíz (\%) }\end{array}$ \\
\hline T1 & 10 & 16 & $117 \pm 0.1^{\mathrm{a}}$ & $114.53 \pm 4.95^{\mathrm{b}}$ \\
\hline T2 & 20 & 16 & $108.1 \pm 3.55^{\mathrm{b}}$ & $116.76 \pm 5.96^{\mathrm{b}}$ \\
\hline T3 & 10 & 30 & $120.6 \pm 0.15^{\mathrm{a}}$ & $118.23 \pm 1.32^{\mathrm{a}}$ \\
\hline T4 & 20 & 30 & $121.4 \pm 0.36^{\mathrm{a}}$ & $119.9 \pm 0.05^{\mathrm{a}}$ \\
\hline
\end{tabular}

${ }^{\text {a }}$ b Representan diferencias estadísticas significativas de acuerdo con la prueba de Tukey $(\mathrm{P}<0.05)$ en los diferentes tratamientos

Determinar el porcentaje de rendimiento en el procesamiento de productos cárnicos es importante, ya que proporciona información útil para la 
selección de proveedores, tomar decisiones en la producción, evaluar aditivos y determinar costos, entre otros (Zamora-Castro et al., 2018).

Esto se explica con la ecuación matemática siguiente relacionada con el rendimiento de la producción de jamón (EC 1), que indicó que los factores tienen efectos positivos y negativos en cuanto al rendimiento del jamón. Cuando se agrega una concentración mayor de almidón o incrementa el tiempo de masajeo, aumenta el rendimiento de jamón:

\section{EC 1}

Rendimiento de jamón cocido $=132.719-$ 0,42619*Tiempo 1,99095* Ligador + 0,0688095*Tiempo*Ligador

También en la Figura 1 se muestra la relación entre el tiempo y la concentración del almidón, donde se observó que al incrementar el tiempo y la concentración del ligador (almidón), aumentó el rendimiento del jamón.

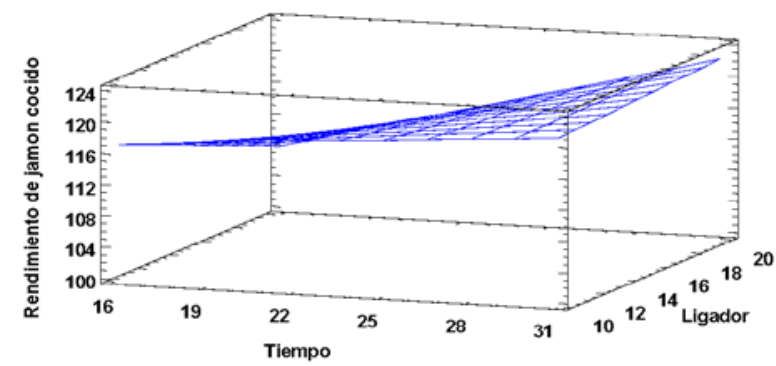

Figura 1. Superficie de respuestas en el rendimiento de jamón con almidón de plátano

Se detectó que los factores de manera individual (concentración de almidón y tiempo), no tienen efecto positivo en el rendimiento del jamón. Sin embargo, al interaccionar los dos factores producen un efecto positivo en el rendimiento.

Es decir, para un mejor rendimiento en la producción de jamón se requiere una combinación de ambos factores: incrementar el tiempo y la concentración de almidón de plátano de manera paralela. Aunque, para conocer el tiempo y la concentración de almidón óptimas, se recomienda una corrida experimental de optimización. Estudios previos, evaluaron el efecto de la sustitución de harina de trigo por almidón de malanga Colocasia esculenta L sobre las pérdidas por cocción y demostraron que todos los tratamientos con almidón de malanga presentaron menores pérdidas por cocción que el producto control (Torres-Rapelo et al., 2014).

Estos resultados se correlacionan con los obtenidos en este trabajo donde el jamón adicionado con almidón de plátano obtuvo un mayor rendimiento que el elaborado con almidón de maíz.

En otro trabajo se evaluó la harina de plátano verde (Musa AAB Horn Plantain Cv. Harto) como sustituto de grasa para productos cárnicos; donde al final, 
recomendaron utilizar de $10 \%$ a $20 \%$ de almidón de plátano en el jamón para disminuir el $20 \%$ de grasas en el producto final (Araya-Quesada et al., 2014). Así, el almidón de plátano ha adquirido importancia en la industria alimentaria como ingrediente o aditivo, por lo que se utiliza frecuentemente como aglutinante, espesante, gelificante, humectante y texturizante.

\section{Capacidad de retención de agua}

La capacidad de retención de agua se define como la habilidad que tiene un producto para retener el agua propia y añadida, cuando se le somete a un esfuerzo mecánico y térmico (Pérez \& Ponce, 2013). Esta propiedad se relaciona con las características de jugosidad, color, y terneza de la carne fresca, así como con el rendimiento en productos cocidos (Soto-Simental et al., 2016). En este trabajo, la ecuación o relación matemática obtenida en la capacidad de retención de agua (CRA) indicó que los factores producen efectos positivos en cuanto a la CRA. Es decir, al incrementar la concentración de almidón o el tiempo de masajeo aumenta la CRA (EC 2).

\section{EC 2}

CRA plátano $=2,06667+0,0333333 *$ Tiempo + 0,0433333*\% de almidón + 0,00166667*Tiempo*\% de almidón

En este trabajo se tuvo un efecto mayor en el jamón adicionado con almidón de plátano en comparación con el adicionado con almidón de maíz.

La Figura 2 muestra que los factores tienen efectos positivos en relación con la capacidad de retención de agua, es decir, al incrementar la concentración de almidón y el tiempo de masajeo aumenta la CRA del jamón, para ambas fuentes de almidón: de plátano o de maíz. Sin embargo, cabe destacar que el jamón cocido con inclusión de almidón de plátano cuadrado presenta un porcentaje mayor de CRA que el jamón cocido con almidón de maíz.
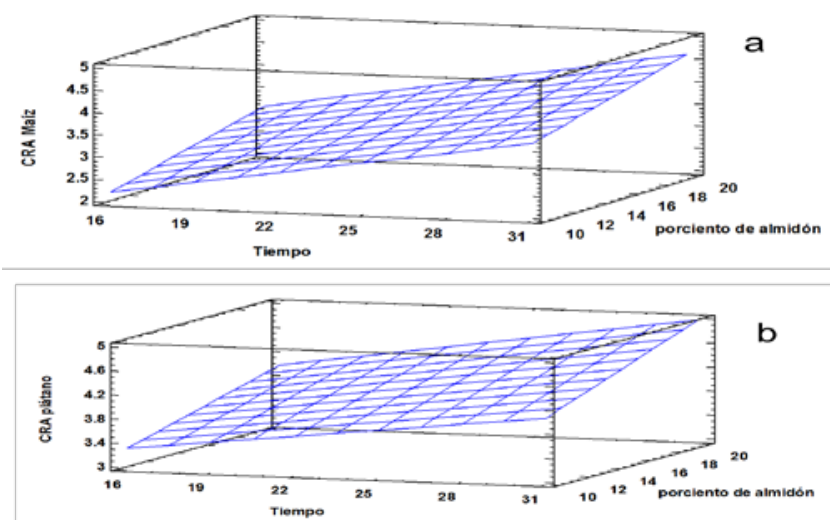

Figura 2. CRA del jamón cocido con almidón de maíz (a) o jamón cocido con almidón de plátano (b) 
En cuanto al $\mathrm{pH}$, se ha citado que está asociado con la capacidad de absorción de agua, la suculencia, el desarrollo del color y la carga microbiológica del producto final (Fernández et al., 2008). El pH de la carne aumenta gradualmente por el incremento de bases volátiles a medida que se suscitan reacciones de proteólisis, descarboxilación y oxidación, entre otras, que en estado avanzado son responsables de su deterioro (Zamora \& Mendoza, 2018). Las características de color, jugosidad y textura, además de otras propiedades como la capacidad de retención de agua y la capacidad de emulsión, dependen en gran medida del $\mathrm{pH}$ de la carne. Por lo que estas variables se consideran los principales indicadores de la calidad de la carne fresca, así como de su aptitud tecnológica para la elaboración de productos cárnicos (Zamora \& Mendoza, 2018).

de 5.7 .

En este trabajo, la carne utilizada como materia prima presentó un $\mathrm{pH}$

Estos resultados se relacionan con el realizado por Fontes et al., (2017), los gránulos de almidón mostraron un buen poder de hinchamiento, lo que demuestra su potencial para el uso en alimentos que requieren retención de agua, como los productos cárnicos y las jaleas. El bajo porcentaje de pérdida de agua durante el almacenamiento y el bajo retroceso obtenido por el estudio del perfil muestran que el almidón es menos propenso a la degradación y la sinéresis, un factor bastante importante cuando se trata del desarrollo de productos que deben mantenerse bajo refrigeración.

\section{Evaluación sensorial}

En el Cuadro 2 se muestra el nivel de agrado del jamón cocido elaborado con la inclusión de almidón de plátano o almidón de maíz.

Donde se encontró que, el almidón de plátano cuadrado obtuvo una mayor aceptación en las categorías de "me gusta” y "me gusta mucho" con un 61.76 $\%$ y $38.23 \%$ respectivamente.

El análisis sensorial genera información que se usa en las decisiones sobre los productos (Ramírez-Camargo et al., 2016).

Cuadro 2. Nivel de agrado de jamón cocido adicionado con almidón de plátano o almidón de maíz

\begin{tabular}{|l|l|l|}
\hline Nivel de agrado & $\begin{array}{l}\text { Almidón de } \\
\text { plátano (\%) }\end{array}$ & $\begin{array}{l}\text { Almidón de } \\
\text { maíz (\%) }\end{array}$ \\
\hline Me gusta mucho & 38.23 & 32,29 \\
\hline Me gusta & 61.76 & 42,01 \\
\hline Ni me gusta ni me disgusta & 0 & 25,68 \\
\hline Me disgusta & 0 & 0 \\
\hline Me disgusta mucho & 0 & 0 \\
\hline
\end{tabular}

La evaluación sensorial es una herramienta de marketing exitosa en el diseño de nuevos productos, tiene gran impacto en la elección de los 
consumidores, ya que ellos determinan su aceptación o rechazo e influyen en las decisiones de compra (Jiménez-Marín et al., 2019).

En la Figura 3 se muestra el nivel de aceptación y preferencia de los jamones cocidos adicionados almidón de plátano o almidón de maíz. Tanto la aceptación como la preferencia fueron mayores en el jamón adicionado con almidón de plátano (53 \% y 37 \% respectivamente).

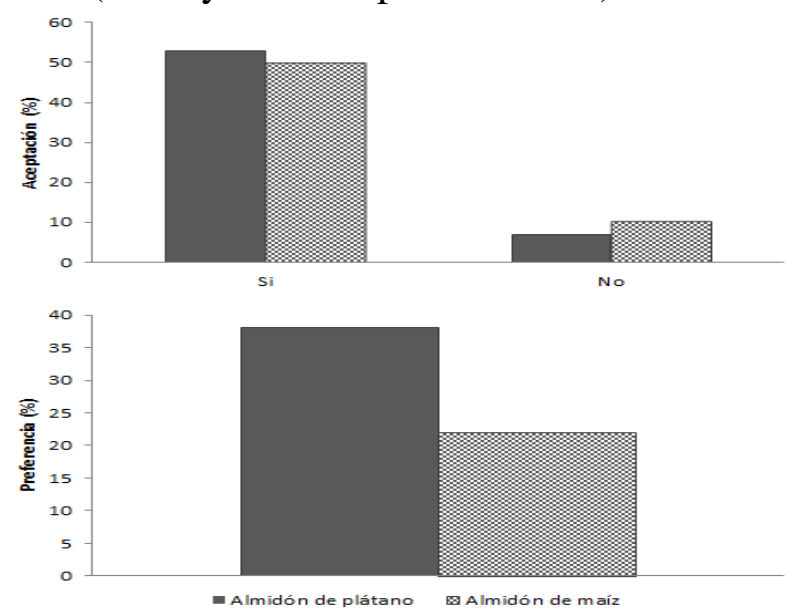

Figura 3. Aceptación y preferencia de jamón cocido adicionado con almidón de plátano o jamón adicionado con almidón de maíz

En un estudio realizado por Contreras-López \& Salvá (2018), encontraron que el almidón de yuca puede ser utilizado para reemplazar la grasa en salchichas. Detectaron comportamiento similar en el patrón sensorial (dureza, cohesividad y masticabilidad) y de textura.

En general, los alimentos procesados son apetecidos por los consumidores, por su costo y características organolépticas (Tofiño-Rivera et al., 2017). Lo que justifica la importancia de proponer aditivos naturales como el almidón de plátano, que tenga propiedades nutritivas del jamón cocido con una mínima inclusión de compuestos químicos.

Lo cual se corroboró en el presente estudio, donde los resultados mostraron que el jamón cocido adicionado con almidón de plátano cuadrado fue el mejor aceptado por las propiedades sensoriales expresadas por los catadores y mejoró el rendimiento. Por lo tanto, puede ser utilizado como un aditivo alimentario para la producción del jamón cocido.

\section{Conclusión}

La concentración de almidón de plátano y el mayor tiempo de masajeo (30 min), registró relación positiva en el rendimiento del jamón cocido. La capacidad de retención de agua aumentó de acuerdo con la concentración de 
almidón y el tiempo de masajeo, independiente del origen del ligante adicionado (almidón de plátano o almidón de maíz).

El jamón cocido adicionado con almidón de plátano cuadrado como agente ligante, fue el más aceptado y mejor evaluado. Por lo que, este almidón puede ser utilizado como un aditivo alimentario para la producción del jamón cocido.

\section{References:}

1. 1.Alao B., Falowo A., Chulayo A. \& Muchenje V. (2017). The potential of animal by-products in food systems: production, prospects, and challenges. Sustainability, 9 (1089): 1-18.

2. Araya-Quesada Y., Morales-Torres A., Vargas-Aguilar P. \& Wexler L. (2014). Potencial tecnológico de harina de plátano verde con cáscara (Musa AAB) como sustituto de grasa para geles cárnicos. Revista del Laboratorio Tecnológico del Uruguay, INNOTEC 9: 50-60.

3. Bohórquez Y., Bonilla M., Pérez I., Quintero S. \& Vargas J. (2017). Caracterización y potencial uso de la raíz achira Canna edulis Ker. Via Innova 4 (1): 89-97.

4. Braña D., Ramírez E., Rubio M., Sánchez A., Torrescano G., Arenas M., Partida J., Ponce E. \& Ríos F. (2011). Manual de Análisis de Calidad en Muestras de Carne. Distrito Federal, (México): Instituto Nacional de Investigaciones Forestales, Agrícolas y Pecuarias, pág. 91.

5. CODEX STAN 96-1981. Norma para el jamón curado cocido (Revisión: 1991, 2014 y 2015). CODEX Alimentarios, normas internacionales de alimentos. FAO, OMS. Pág. 5.

6. Chel-Guerrero L., Barbosa-Martín E., Martínez-Antonio A., González-Mondragón E. \& Betancur-Ancona D. (2016). Some physicochemical and rheological properties of starch isolated from avocado seeds. International Journal of Biological Macromolecules 86: 302-308.

7. Compendio Estadístico, 2021. Compendio Estadístico de la industria cárnica. Consejo Mexicano de la Carne A.C. (COMECARNE) Pág. 97.

8. Contreras-López E. \& Salvá B. (2018). Caracterización sensorial de hamburguesa de llama con cáscara de sanky. Revista investigación Altoandina 20 (2): 155-168.

9. Contreras-Pérez RK., de la Torre-Gutiérrez L., González-Cortés N. \& Jiménez-Vera R. (2018). Caracterización funcional de almidones de plátano cuadrado Musa balbisiana Colla. European Scientific Journal 14 (30): 82-97.

10. Fernández R.M.V., Morales M.J.C., Montiel C.A. \& Mendívil M.M. (2008). Composición proximal y atributos sensoriales de jamón 
preparado con tres niveles de inyección de salmuera. Biotecnia, X (3): 48-58.

11. Fontes S., Cavalcanti M., Candeia R. \& Almeida E. (2017). Characterization, and study of functional properties of banana starch green variety of Mysore Musa AAB - Mysore. Food Science and Technology 37 (2): 224-231.

12. García-Solís S.E., Bello-Pérez L.A., Agama-Acevedo E. \& FloresSilva P.C. (2018). Plantain flour: A potential nutraceutical ingredient to increase fiber and reduce starch digestibility of gluten-free cookies. Starch-Stärke 70 (1700107): 1-5.

13. Hernández I., Martínez M., Contreras R. \& Pérez R. (2017). Extracción de almidón por el método seco en plátano macho, cuadrado y castilla. Revista de Simulación y Laboratorio 4 (13): 1-7.

14. Jaimes J., Torres J. \& Severiche C. (2015). Análisis de la calidad de un producto cárnico escaldado elaborado con harina de Prosopis juliflora. Ingenium 9 (26): 21-28.

15. Jiménez-Marín, G., Bellido-Pérez, E., \& López-Cortés Á. (2019). Marketing sensorial: el concepto, sus técnicas y su aplicación en el punto de venta. Vivat Academia, (148), 121-147.

16. Julio L., Montero P. \& Acevedo D. (2015). Calidad y aceptabilidad de chorizos formulados con plasma sanguíneo bovino y pasta de ajonjolí. Información Tecnológica 26 (3): 25-32.

17. Luna J.J. \& Guerrero-Beltrán J.A. (2013). Caracterización de jamones adicionados con pastas residuales de la extracción mecánica de aceite de frutos secos. Scientia Agropecuaria 4: 77-88.

18. Marrugo-Ligardo Y., Ramirez-Martinez D., Trujillo-Ramos N., Severiche-Sierra C. \& Jaimes-Morales J. (2017). Development of a scalded meat product added with modified bean starch Zaragoza Phaseolus lunatus red variety. Contemporary Engineering Sciences 10 (39): 1473-1483.

19. NOM-213-SSA1-2018, Norma Oficial Mexicana. Productos y servicios. Productos cárnicos procesados y los establecimientos dedicados a su proceso. Disposiciones y especificaciones sanitarias. Métodos de prueba.

20. Pérez C.M.L. \& Ponce A.E. (2013) Manual de prácticas de laboratorio. Tecnología de Carnes. Primera edición. Universidad Autónoma Metropolitana, pp 110.

21. Porcicultura.com (2020). Mercado de las carnes frías en México; un sector en incertidumbre por el Covid-19. Redacción Porcicultura.com. Noticia; 31 de julio, 2020. Consultado: 7 junio, 2021. 
https://www.porcicultura.com/destacado/Mercado-de-las-carnesfrias-en-Mexico\%3B-un-sector-en-incertidumbre-por-elCovid\%7C19

22. PROFECO (2016). El Laboratorio Profeco reporta: Jamón. Revista del Consumidor, pág. 40-51.

23. Ramírez-Camargo E., Marulanda A. \& Orrego J. (2016). Desarrollo de una mezcla de fibras y almidones como reemplazante de grasa para productos de pasta fina tipo salchicha. Información Tecnológica 27 (1): 41-52.

24. Soto-Simental S., Valera-Quezada E., Hernández-Chavez J.F., Güemes-Vera, N. \& Ayala-Martínez M. (2016). Efecto de grasa, agua añadida, carragenina y fosfatos en un producto emulsionado con carne de carpa (Cyprinus carpio). Agrociencia, 50 (4), 413-427.

25. Ssonko U. \& Muranga F. (2017). Partial characterization of starches from major banana (matooke) cultivars grown in Uganda. Food Science and Nutrition (5): 1145-1153.

26. Tofiño-Rivera A., Ortega-Cuadros M., Herrera-Hinojosa B., FragosoCastilla P. \& Pedraza-Claros B. (2017). Conservación microbiológica de embutido cárnico artesanal con aceites esenciales Eugenia caryophyllata y Thymus vulgaris. Biotecnología en el Sector Agropecuario y Agroindustrial, Edición Especial (2): 30-41.

27. Toledo O. (2019). El porcentaje y tipo de almidón (papa o trigo) afectan la textura instrumental de batidos cárnicos reducidos en grasa. NACAMEH, 13 (1): 1-10.

28. Torres-Rapelo A., Montero-Castillo, P. \& Julio-González, L. 2014. Utilización de almidón de malanga Colocasia esculenta L. en la elaboración de salchichas tipo Frankfurt. Biotecnología en el Sector Agropecuario y Agroindustrial, 12 (2): 97-105.

29. Zamora-Castro, J E., Illescas-Chávez E., Hernández-Zárate G. \& Lunadimas, M. (2018). Uso de ingredientes funcionales no cárnicos en la elaboración de jamón cocido artesanal. Revista de Operaciones Tecnológicas, 2-6: 24-30.

30. Zamora R., \& Mendoza L del R. (2018). Calidad de la carne del ganado vacuno. Revista Observatorio de la Economía Latinoamericana. https://www.eumed.net/rev/oel/2018/04/calidad-carne-ecuador.html. 